\title{
Editorial
}

\section{Chromatographic technique to support ADMET\&DMPK in early drug discovery}

\author{
Klara Valko ${ }^{1,2}$
}

\author{
Associate Editor ADMET \& DMPK \\ ${ }^{1}$ Bio-Mimetic Chromatography Ltd, Business \& Technology Centre, Stevenage, SG1 2DX United Kingdom \\ ${ }^{2}$ Department of Biological and Pharmaceutical Chemistry, UCL School of Pharmacy London WC1N 1AX United Kingdom \\ E-mail: K.Valko@ucl.ac.uk and Klara_Valko@bio-mimetic-chromatography.com; Tel.: +44 7521989558
}

The drug discovery process usually involves the discovery of a potent new chemical entity which has shown some activity on a target that is believed to be relevant in a certain type of disease. However, the structure and property of these putative drug molecules may have to be modified in order to ensure that they are able to exert the desired in vivo pharmacological effect. The candidate molecules usually have to be absorbed from the gastrointestinal system and therefore they must show appropriate solubility and permeability to be able to reach the target enzyme in vivo with a sufficient free biophase concentration at the site of action. This means that the physicochemical properties and the protein and phospholipid binding has to be optimized in order to achieve the desired in vivo ADMET and DMPK profile. The ADME studies usually require animal experiments or the use of biological samples, for example tissues which is expensive and involves time-consuming procedures. New technologies and approaches can accelerate this process.

HPLC methodology is well established and used throughout the drug discovery process for analytical measurements of a compound's purity and concentration in various biological samples such as blood, plasma and tissues. During the HPLC separation process, the compounds distribute between the mobile and the stationary phases. As different compounds have a different interaction with the stationary phase that is modified by the mobile phase, they move at a different speed through the chromatographic system resulting in different retention times. As these retention times are characteristic of the compound and the HPLC system and can be related to a compound's dynamic distribution coefficient, they can be used to describe the properties of the compounds. When biomimetic stationary phases are used during chromatography, such as albumin, glycoprotein, and phospholipids, the calibrated retention times can be converted to binding constants representing these body components. These data can then be used to estimate the compound's in vivo distribution behavior.

In view of the important roles of HPLC methodology, ADMET \& DMPK devoted a special issue to cover chromatographic technique in early drug discovery. Contributions were invited and received from several researchers who are well respected in this field to enlighten the readers of the more recent developments and applications in chromatography and in separation science as applied in support of drug discovery. This is presented as both original scientific papers describing the original contributions or review articles.

The application of chromatography to predict skin penetration of compounds by Marti Rosés and his 
team at University of Barcelona, Spain is described [1]. The application of pressure assisted Microemulsion Electro-kinetic Chromatography for lipophilicity determination has also been described by the same research group in a second contribution [2].

Automated measurements using biomimetic chromatography in support of drug discovery programme research at GSK are reviewed by Shenaz Bunally and Rob Young [3]. Similar efforts in Pfizer has been reviewed by Gilles Goetz and Marina Shalajeva in their contributions including their recent application of supercritical chromatography to measure the embedded polar surface area (EPSA) of molecules that could be related to their permeability [4].

Klara Valko and co-workers from Genervon Biopharmaceuticals LLC (California, USA) and Bicycle Therapeutics (Cambridge, United Kingdom) have investigated the application of biomimetic chromatography to estimate lipophilicity, protein and phospholipid binding of new modalities such as linear and cyclic peptides as potential therapeutics [5]. The in vivo distribution behavior, cellular accumulation and brain penetration have been estimated using biomimetic HPLC measurements for GM6, a linear peptide discovered by Genervon Biopharmaceuticals, that has shown therapeutic potential in vivo for Amyotrophic Lateral Sclerosis (ALS) [6].

Oleg Krokhine and his research group at the University of Manitoba, Canada have demonstrated the possibility to predict peptide interaction with Immobilized Artificial Membranes using over 30 thousand peptides from tryptic digest to estimate their phospholipid binding and have compared these interactions with traditional reversed-phase chromatography that is most widely used for lipophilicity measurements [7].

Several papers belonging to the chromatography thematic issue will be published in the next issue of ADMET and DMPK including the papers of Ciesla et al. [8], Tsopelas et al. [9] and Caron et al. [10].

Lukasz Ciesla and his colleagues at the University of Alabama, USA have presented a new and innovative application of the immobilized artificial membrane stationary phase to bind membrane receptors on the surface called cellular membrane affinity chromatography (CMAC) that can enable the "fishing out" of potent molecules from complex mixtures [8].

Fotios Tsopelas, Chrysanthos Stergiopoulos and Anna Tsantili-Kakoulidou have presented a comprehensive review article on the application of Immobilized Artificial Membrane Chromatography (IAM) in support of the drug design and discovery process [9].

Giulia Caron and her colleagues at the University of Torino, Italy have presented a review article about the block relevance approach which they developed to identify structure-property correlations that significantly enhanced the design of molecules with the right ADMET and DMPK properties [10].

The guest editor would like to thank all the contributors for their tremendous efforts made in presenting their work so lucidly. It is hoped that readers will be pleased to read these contributions and find them useful in their search for new drugs that will help to cure diseases and improve the quality of life of humans.

\section{References}

[1] S. Soriano-Meseguer, E. Fuguet, A. Port, Martí Rosés. Estimation of skin permeation by liquid chromatography. ADMET and DMPK 6 (2018) 140-152.

[2] X. Subirats, L. Redón, M. Rosés. Lipophilicity determination of acidic compounds: MEEKC as a reliable high-throughput methodology. ADMET and DMPK 6 (2018) 153-161. 
[3] S. Bunally, R.J. Young, The role and impact of high throughput biomimetic measurements in drug discovery. ADMET and DMPK 6 (2018) 74-84.

[4] G.H. Goetz, M. Shalaeva. Leveraging chromatography based physicochemical properties for efficient drug design. ADMET and DMPK 6 (2018) 85-104.

[5] K. Valko, G. Ivanova-Berndt, P. Beswick, M. Kindey, D. Ko, Application of biomimetic HPLC to estimate lipophilicity, protein and phospholipid binding of potential peptide therapeutics. ADMET and DMPK 6 (2018) 162-175.

[6] K. Valko, M. Kindy, J. Evans, D. Ko, In vitro biomimetic HPLC and in vivo characterisation of GM6, an endogenous regulator peptide drug candidate for amyotrophic lateral sclerosis. ADMET and DMPK 6 (2018) 176-189.

[7] D. Gussakovsky, H. Neustaeter, V. Spicer, O.V. Krokhin. Peptide retention time prediction for immobilized artificial membrane phosphatidylcholine stationary phase: method development and preliminary observations. ADMET and DMPK 6 (2018) 190-199.

[8] C. Stephen, A. El Omri, L. Ciesla. Cellular membrane affinity chromatography (CMAC) in drug discovery from complex natural matrices. ADMET and DMPK 6 (2018) doi: http://dx.doi.org/10.5599/admet.535.

[9] F. Tsopelas, C. Stergiopoulos, A. Tsantili-Kakoulidou. Immobilized artificial membrane chromatography: from medicinal chemistry to environmental sciences. ADMET and DMPK 6 (2018) to be published.

[10] G. Ermondi, G. Caron. Block relevance (BR) analysis and polarity descriptors in property-based drug design. ADMET and DMPK 6 (2018) http://dx.doi.org/10.5599/admet.532. 